Disputar la autoridad (o el debate sobre la autoridad etnográfica y las perspectivas epistemológicas en pugna)

Mariana Caviglia

Tram[p]as de la comunicación y la cultura (N. $\left.{ }^{\circ} 84\right)$, e039, 2019

ISSN 2314-274X | https://doi.org/10.24215/2314274xe039

http://perio.unlp.edu.ar/ojs/index.php/trampas

FPyCS | Universidad Nacional de La Plata

La Plata | Buenos Aires | Argentina

\title{
DISPUTAR LA AUTORIDAD
}

(O EL DEBATE SOBRE LA AUTORIDAD ETNOGRÁFICA

\section{Y LAS PERSPECTIVAS EPISTEMOLÓGICAS EN PUGNA)}

\author{
DISPUTE AUTHORITY
}

(OR THE DEBATE ON ETHNOGRAPHIC AUTHORITY

AND THE EPISTEMOLOGICAL PERSPECTIVES IN CONFLICT)

\author{
Mariana Caviglia \\ mcaviglia@perio.unlp.edu.ar \\ https: / / orcid.org/0000-0003-2181-5957 \\ Instituto de Estudios Comunicacionales en Medios, \\ Cultura y Poder "Aníbal Ford" (INESCO) \\ Universidad Nacional de La Plata | Argentina
}

\begin{abstract}
Resumen
La autora recupera el debate sobre la autoridad etnográfica que se produjo en la antropología a partir de las corrientes posmodernas que redujeron la cultura, la etnografia y la reflexividad a la textualidad, y aborda los principales cuestionamientos a los supuestos en los que se funda esta concepción y la problematización sobre las formas de construcción de la autoridad, desde la asunción de la etnografía como perspectiva y método de conocimiento.
\end{abstract}

Abstract

The authoress recovers the debate around the ethnographic authority that occurred in anthropology from the postmodern currents that reduced culture, ethnography and reflexivity to textuality, and addresses the main questions to the assumptions on which this conception is based and the problematization on the forms of construction of authority, from the assumption of ethnography as a perspective and method of knowledge.

Palabras clave | autoridad etnográfica, etnografia, reflexividad, cultura

Keywords | ethnographic authority, ethnography, reflexivity, culture 


\title{
DISPUTAR LA AUTORIDAD
}

(O EL DEBATE SOBRE LA AUTORIDAD ETNOGRÁFICA

Y LAS PERSPECTIVAS EPISTEMOLÓGICAS EN PUGNA)

\author{
Por Mariana Caviglia
}

En el campo de la antropología, las perspectivas denominadas posmodernas pusieron en discusión una serie de cuestiones relacionadas con el modo en que se construye la autoridad etnográfica, mediante el análisis de las maneras en que la etnografia se produce y se presenta, y los diversos estilos narrativos que se ponen en juego, a partir del supuesto de que detrás de los mismos se esconde una relación de dominación y de imposición y, por lo tanto, una forma de autoritarismo hacia aquellos otros que la antropologia, desde una perspectiva relativista, buscaria comprender en sus propios términos, a través de una intervención ético-política que supondría relativizar la propia racionalidad y reconocer la existencia de otras racionalidades, de otros "puntos de vista", los nativos, y que, en la tradición antropológica, se encuentra asociada al concepto de cultura.

En El antropólogo como autor (1989), Clifford Geertz sostuvo que el problema de la distancia entre la experiencia del trabajo de campo y la producción, y la presentación autorizada de la etnografia se resolvió a partir de crear la ilusión -mediante la escritura etnográfica y el uso de ciertas estrategias retóricas persuasivas- de que los/as antropólogos/as realmente han "estado alli». En otras palabras, que la autoridad etnográfica no se sustenta ni en la amplitud de las descripciones factuales ni en la argumentación teórica o en la solidez conceptual, sino en la habilidad de persuadir al lector, a través de la escritura, de que el "milagro invisible" del encuentro con el otro realmente ha ocurrido. 
En este sentido, el convencimiento sobre la autoridad del conocimiento se conseguiría con estrategias literarias que irian más allá de su modo de producción. Al mismo tiempo, la autoridad del discurso del/la etnógrafo/a (en tanto autor/a) se relacionaría, según Geertz (1989), con una cuestión de poder: "En nuestra ingenua disciplina [...] importa aún mucho quién habla" (p. 16). La postura se reafirma con la referencia a Bronisław Malinowski, quien -ironiza Geertz (1989)-, aun cuando su aparato teórico, "en otro tiempo una orgullosa torre, yace hoy en gran medida en ruinas (...) sigue siendo el etnógrafo por antonomasia" (p. 14), y con la alusión a la publicación póstuma de su diario.

De este modo, Geertz (1989) ponía en cuestión el realismo etnográfico que se sostenía sobre la autoridad del/la etnógrafo/a en tanto "testigo objetivo". Tras la crítica al paradigma realista, se abría paso el paradigma interpretativo, la antropología se constituía en ciencia de la cultura y su tarea se orientaba a interpretar las interpretaciones que sobre su propia realidad realizaban aquellos otros que se avocaba a estudiar. La cultura, y por tanto la etnografia, eran entendidas, entonces, como un texto.

La imposibilidad de dar cuenta "objetivamente" de otras realidades desde el punto de vista nativo (donde "objetivamente" significa encontrar fundamentos empíricos en los datos de la realidad de ese punto de vista, de acuerdo con los patrones de la ciencia occidental), había sido apuntada por Peter Winch (1994) en Comprender una sociedad primitiva, a propósito de su crítica a Edward Evan Evans-Pritchard. Allí, el filósofo británico ponía en tela de juicio el contraste entre las creencias de los Azande ${ }^{1}$ y las creencias de las sociedades modernas que Evans-Pritchard había señalado en Brujería, magia y oráculos entre los Azande (1976 [1937]).

Básicamente, Winch (1994) cuestionaba que Evans-Pritchard (1976 [1937]) -quien pretendia entender el mundo desde el punto de vista nativo, pero se preguntaba cómo hacerlo y cómo explicarlo, precisamente, cuando los antropólogos occidentales no aceptaban ciertas creencias nativas- afirmara que las creencias de los Azande eran lógicas aunque equivocadas, puesto que no eran susceptibles de fundamentación empírica (es decir, que eran falsas al no estar en concordancia con la realidad "objetiva", mientras que las nuestras serian verdaderas). 
La tesis de Winch (1994), acerca de que lo que determinaba lo que estaba en concordancia con la realidad no era la verificación empírica sino los juegos del lenguaje -a través de los que se constituye intersubjetivamente la realidad-, enunciaba que no existía realidad "objetiva" exterior al lenguaje y a la cultura, es decir, que las creencias azande eran falsas según nuestras concepciones de la realidad. Por tanto, al intentar comprenderlas imponemos nuestras concepciones y el modelo de verificación empírica propios de la ciencia occidental, que no es otra cosa que un juego más del lenguaje.

Así, aquella noción de la práctica etnográfica en la cual esta se disponía a la búsqueda de universales quedaba descartada, y aquel proyecto en el cual la etnografia se constituía en traductora universal de culturas, de distintas racionalidades para la sociedad -dado que al estar la realidad contenida en el lenguaje no habría traducciones válidas entre culturas sin que esto supusiera traicionar su lógica propia-, también se encontraba imposibilitado, a no ser que los/as antropólogos/as se deshicieran del modelo científico y adoptaran el principio del relativismo cognoscitivo con la intención de traducir el "punto de vista nativo".

El planteo de Winch (1994) refiere, en última instancia, a la idea de que no existe una racionalidad común y compartida y, de este modo, a la inconmensurabilidad de las culturas (de ahí que señalara la existencia de ciertos universales -el nacimiento, el sexo y la muerte- que serian el objeto de la antropología y en torno a los cuales esta podría adoptar una perspectiva relativista, es decir, relativizar su propia racionalidad y reconocer la existencia de otras racionalidades en torno a estas experiencias humanas compartidas).

Ahora bien, sobre la base de estos supuestos -la inexistencia de una realidad exterior al lenguaje; la incapacidad de la ciencia occidental para comprender otros mundos sin distorsionarlos; la imposibilidad de dar cuenta del "punto de vista nativo", sin imponer las propias categorias a partir de las cuales el mismo sería aprehendido; el hecho de que la posibilidad de penetrar o de ser penetrados realmente por otras culturas, como escribe Geertz (1989), no sea más que una ilusión, etc.- fue que antropólogos como James Clifford (1992 [1988]) y Stephen Tyler (1992 [1986]), entre otros posmodernos, desarrollaron una perspectiva según la cual las etnografias no serian otra cosa que textos, narrativas, relatos o, en términos de Marilyn Strathern (1987), "ficciones 
persuasivas", dada la imposibilidad de reflejar la realidad y debido a que en la capacidad de persuadir se constituiria, precisamente, su autoridad.

Desde la mirada de estos/as autores/as, los cuestionamientos y las criticas al empirismo parecen transformarse en puro análisis y en crítica textual. Es decir, la crítica al empirismo científico se convierte en una concepción simplista de la etnografia que parece ser comprendida y absolutizada como literatura. La incapacidad de reflejar una realidad "objetiva" se transforma en pura experiencia subjetiva reflejada en el texto, de modo que el problema, o mejor, el objeto de la etnografia, pasa a ser cómo relatar convincentemente la experiencia subjetiva en el campo.

El/la etnógrafo/a que antes se pretendia capaz de representar la cultura nativa se transforma en aquel/lla que le posibilita a esta construir una representación dialógica (que, en tanto tal, se supone más democrática, veraz y compartida). Y su autoridad como "testigo objetivo", en la cual se fundaba el realismo etnográfico, se transforma en una autoridad proveniente de la capacidad de construir una ficción persuasiva de esa experiencia subjetiva.

Ahora bien, aunque es cierto que la experiencia se expresa en el relato o -como trató de poner sobre el tapete Geertz (1989) ante cierta ingenuidad literariaque hay un sujeto histórico que se expresa en la narración, también es cierto que desde el enfoque de estos/as autores/as posmodernos/as esto no parece ser problematizado, y que lo que se pone en cuestión y se constituye como problema no es la propia experiencia (que, por otra parte, tampoco es el objeto de la antropología) sino cómo esta es narrada. Toda la reflexividad queda reducida, entonces, a la experiencia del/la autor/a y a cómo autorizar el relato a través de la escritura (Clifford, 1992 [1988]; Geertz, 1989).

Sin embargo, la etnografía no es apenas el producto de la experiencia de quien investiga, sino que se produce al interior de un campo, condicionada por relaciones políticas e institucionales específicas, en diálogo con todo un universo de investigación, en función de trayectorias personales y académicas particulares, etc., y, por ende, no puede reducirse a la mera expresión de una subjetividad aislada en un texto, puesto que la etnografia no es nunca tan solo el producto de un trabajo de campo (trabajo de campo que, además, tampoco es problematizado como condicionante y como configurador de la obra) y, menos aún, es tan solo un texto y la presencia de un/a autor/a a la cual 
todo queda subordinado. Asimismo, sostener que la etnografia es un género textual supone negar que es también una perspectiva de conocimiento y un método, en suma, una disciplina científica.

Podriamos preguntarnos si al interior del debate posmoderno la ausencia de consideración y de problematización de estas cuestiones no llevó a los/as autores/as así denominados a imponer sus propias categorizaciones sobre la construcción de la autoridad etnográfica, si no hay allí una ausencia de relativización de esa categoría y si no fueron leídas fuera de contexto las intenciones de otros/as autores/as, en este caso, las de los/as antropólogos/as clásicos/as. Pues si bien es claro que toda obra apela a cierta autoridad, desde este punto de vista pareciera inferirse que todo/a autor/a de una etnografia tiene la intención de construir deliberadamente una autoridad etnográfica específica, que puede leerse en los diversos tipos de estrategias literarias o de recursos de escritura presentes en el texto (asi lo infiere Geertz [1989] en "Estar allí. El antropólogo y la escena de la escritura" y lo analiza claramente Clifford [1988] en "Sobre la autoridad etnográfica»).

No es que la construcción de autoridad no exista, sino que un interrogante posible es si se trata del producto de una voluntad tan deliberada y conciente (o, al menos, determinada absolutamente por esa intencionalidad) o si la mayor parte del tiempo, es producto de que las etnografias no son exclusivamente el resultado de una experiencia subjetiva, sino que se producen en contextos específicos, para determinados/as lectores/as, y en un universo que tiene sus reglas y sus convenciones, y que las moldea y las condiciona.

Tal vez, donde Geertz o Clifford leyeron que ciertos/as antropólogos/as quisieron remarcar que "estuvieron alli" eso deba leerse no como una simple forma de construir autoridad a partir de una estrategia literaria sino, también, como el resultado de una investigación etnográfica que no se define meramente por ser un texto sino un método que proviene de un enfoque particular y que consiste, ni más ni menos, en haber estado allí, porque ese texto es, entre otras cosas, el producto de un método que en efecto se define por haber estado en ese otro mundo.

De igual modo, si Malinowski se planteaba, en palabras de Geertz (1989), "cómo autorizar una presentación digna de fe en los hechos" (p. 93), es posible que no estuviese pensando en autorizarla exactamente del modo en que él 
la da por supuesto ni en construir la autoridad en la forma que le atribuye, en un momento completamente diferente de la historia de la antropología y en un contexto de producción absolutamente otro.

Esa falta de contextualización y de historización del debate descuida que la problematización sobre las formas de construcción de la autoridad en una disciplina constituye, ante todo, la expresión de un conflicto de autoridades, una disputa por quién tiene la autoridad al interior de ese campo disciplinario (tal vez aquí se halle otra clave de lectura de aquella afirmación de Geertz [1989] sobre la importancia de quién habla).

Asimismo, el planteo posmoderno de que la legitimidad y la autoridad se basan en "estar ahí" parece suponer que el público de las etnografias -aquellos/as lectores/as que autorizarian la obra- se extiende mucho más allá de la comunidad antropológica a la que las etnografias están, ante todo, dirigidas. Las etnografias parecen ser, así, textos fuera de contextos mientras que estos últimos condicionan el público al que las obras se dirigen y, al mismo tiempo, pueden crear la necesidad de autorizar las etnografias frente a diversos públicos en diversos momentos históricos de la disciplina.

En tanto, la pretendida construcción dialógica de las etnografias, planteada por antropólogos como Tyler (1992 [1986]), la supuesta capacidad que se autoatribuyen de darle al otro, a partir del diálogo, su propia voz, antes silenciada, y de permitirle expresarse libremente, no deja de ser una ilusión, puesto que continúa tratándose de un acto de poder y sigue siendo el/la etnógrafo/a quien ejerce el control. Un control que es propio, inherente a esa relación.

Con todo, esta concepción de la etnografia como texto en sí mismo, que se estructuró en un momento de descenso de la antropología británica y de ascenso de la norteamericana, al tiempo que se producía una aproximación a los estudios culturales y literarios, encuentra sus fundamentos en una concepción de la cultura que, en tanto texto, narrativa o ficción, la convierte en un dato objetivado, esencial, homogéneo y reificado. Pues desde este enfoque, la cultura es singular y ahistórica.

Sin embargo, es posible comprender la cultura, junto con Christoph Brumann (1999), como una abstracción y a las etnografias como construcciones de abstracciones que se realizan, valga la redundancia, abstrayendo de la realidad 
aquello que aparece como regular. En este sentido, se trataria, antes que de un texto, de una conceptualización que transmite sentidos reconocibles para los/as integrantes de una comunidad específica y que se produce no a partir de un ingreso desprejuiciado del/la etnógrafo/a al campo sino a partir de un modelo basado empíricamente, constituido por conceptos claros y operativos que permiten recortar la realidad y construir nexos, como así también interpretarla, reconociendo relaciones entre datos dispersos, entre ciertos indicios y el modelo.

En este sentido, la autoridad etnográfica proviene de la solidez de un modelo empíricamente informado, de la conmensurabilidad entre esa conceptualización y las otras conceptualizaciones producidas por aquellos/as a quienes la etnografia se dirige. "Estar ahí" no es sinónimo de tal autoridad. Es el método que se desprende de una perspectiva de conocimiento que, desde un enfoque que busca comprender los fenómenos socioculturales, se define por incluir las perspectivas de sus miembros (en plural) poniéndolas en contexto (es decir, interpretando los puntos de vista nativos). Y, desde allí, "estar ahí» es siempre una forma de ampliar el universo discursivo y de traducir las experiencias en algo significativo, haciendo posible darle sentido al universo abstracto de la teoría.

\section{Referencias}

Brumann, C. (1999). Writing for Culture: Why a Successful Concept Should not be Discarded. Current Anthropology, 4O(Suppl), S1-S27. https://doi.org/10.1086/200058

Clifford, J. (1992) [1988]. Sobre la autoridad etnografica. En C. Reynoso (Comp.), El surgimiento de la antropología posmoderna. (pp. 141-170). Barcelona, España: Gedisa.

Evans-Pritchard, E. E. (1976) [1937]. Brujería, magia y oráculos entre los Azande [Witchcraft, Oracles and Magic Among the Azande]. Barcelona, España: Anagrama.

Geertz, C. (1989). El antropólogo como autor. Barcelona, España: Paidós. 
Strathern, M. (1987). Out of Context: The Persuasive Fictions of Anthropology. Current Anthropology, 28(3). Recuperado de https: / / www.journals.uchicago.edu/doi/pdf/10.1086/203527

Tyler, S. (1992) [1986]. La etnografia posmoderna: de documento de lo oculto a documento oculto. En C. Reynoso (Comp.), El surgimiento de la antropología posmoderna (pp. 297-314). Barcelona, España: Gedisa.

Winch, P. (1994). Comprender una sociedad primitiva. Barcelona, España: Paidós.

Nota

1 Los Azande son un pueblo del centro-norte de África. 